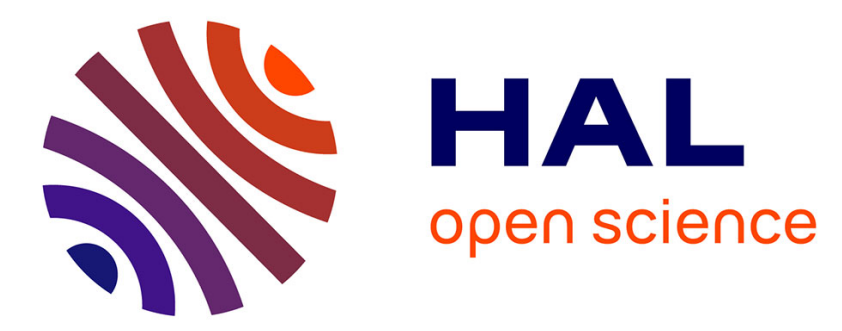

\title{
Sub-femtosecond electron transport in a nanoscale gap
}

Markus Ludwig, Garikoitz Aguirregabiria, Felix Ritzkowsky, Tobias Rybka, Dana Codruta Marinica, Javier Aizpurua, Andrei Borisov, Alfred Leitenstorfer, Daniele Brida

\section{- To cite this version:}

Markus Ludwig, Garikoitz Aguirregabiria, Felix Ritzkowsky, Tobias Rybka, Dana Codruta Marinica, et al.. Sub-femtosecond electron transport in a nanoscale gap. Nature Physics, 2020, 16 (3), pp.341345. 10.1038/s41567-019-0745-8 . hal-02993162

\section{HAL Id: hal-02993162 \\ https://hal.science/hal-02993162}

Submitted on 9 Nov 2020

HAL is a multi-disciplinary open access archive for the deposit and dissemination of scientific research documents, whether they are published or not. The documents may come from teaching and research institutions in France or abroad, or from public or private research centers.
L'archive ouverte pluridisciplinaire HAL, est destinée au dépôt et à la diffusion de documents scientifiques de niveau recherche, publiés ou non, émanant des établissements d'enseignement et de recherche français ou étrangers, des laboratoires publics ou privés. 


\title{
Sub-femtosecond Electron Transport in a Nanoscale Gap
}

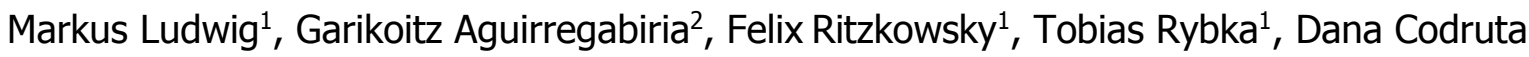 \\ Marinica ${ }^{3}$, Javier Aizpurua ${ }^{2}$, Andrei. G. Borisov ${ }^{3}$, Alfred Leitenstorfer ${ }^{1}$, Daniele Brida ${ }^{1,4}{ }^{*}$ \\ ${ }^{1}$ Department of Physics and Center for Applied Photonics, University of Konstanz, D-78457 Konstanz, Germany \\ ${ }^{2}$ Centro de Física de Materiales, Centro Mixto CSIC-UPV/EHU and Donostia International Physics Center (DIPC), \\ 20018 Donostia-San Sebastián, Spain \\ ${ }^{3}$ Institut des Sciences Moléculaires d'Orsay - UMR 8214, CNRS-Université Paris Sud, Bâtiment 520, Orsay Cedex \\ 91405, France. \\ ${ }^{4}$ Physics and Materials Science Research Unit, University of Luxembourg,162a avenue de la Faïencerie, L-1511 \\ Luxembourg, Luxembourg \\ * e-mail: daniele.brida@uni.lu
}

\begin{abstract}
We employ near-infrared single-cycle pulse pairs to drive interferometric autocorrelations of the ultrafast current produced by optical field emission at the nanogap of a single plasmonic nanocircuit. This highly nonlinear process depends fully on the precise temporal field profile of the optical driving pulse. Current autocorrelations are acquired with sub-femtosecond temporal resolution as a function of both pulse delay and absolute carrierenvelope phase. In this way, we study the ultrafast dynamics of electronic nanotransport. Quantitative modelling of the experiments enables us to monitor the spatio-temporal evolution of the electron density and currents induced in the system and to elucidate the physics underlying the electron transfer driven by strong optical fields in plasmonic gaps. Especially, we clarify the interplay between carrier-envelope phase of the driving pulse, plasmonic resonance and quiver motion.
\end{abstract}

Femtosecond pulses consisting of only a few cycles of the optical carrier wave [1] have enabled breakthrough opportunities for the direct manipulation of electrons in condensed matter based on nonlinear phenomena as well as in free space via linear acceleration $[2,4,5,6,7,8,9,21,24,25,27,28,29,30,31,32,34]$. The traditional conditions for such control experiments required extreme pulse intensities at low repetition rates, imposing substantial difficulties for the extraction of the dynamics of individual charge carriers. Recently, several works paradigmatically changed the approach to exploit optical phenomena driven by strong fields by employing pulses with minute energy content at high repetition rates, thus allowing for generation and manipulation of photoelectrons in nanoscale systems $[5,16,33]$. 
In this context, single-cycle pulses with an energy of only a few picojoules may result in extremely nonlinear optical phenomena within a few-nanometer gap between the tips of an optical antenna $[13,14,15,16,23,36]$. The strong electrical bias provided by the field contained in the ultrashort optical pulses was harnessed to drive tunneling and ballistic acceleration of electrons generating a current with $\mathrm{PHz}$ bandwidth [16]. This non-perturbative process is fully coherent with the driving radiation and occurs within a half-cycle of the near-IR carrier wavelength [16]. Here, by performing interferomeric autocorrelation measurements with two identical replicas of truly single-cycle driving pulses, we directly access the attosecond dynamics of the optical field emission and electron transport in plasmonic gaps. Controllable variation of the carrier-envelope phase (CEP) of the pulses enriches the experimental acquisition of the interferometric traces by an additional variable that allows a complete insight into the relationship between the photo-emitted electrons and the accelerating field. Time-dependent density functional theory (TDDFT) calculations of the optical field emission and subsequent acceleration in the plasmonic gap accurately reproduce the experimental results. In this way, we elucidate the underlying microscopic phenomena, including visualization of the evolution of electron currents in time and space and as a function of the CEP of the driving pulses.

Figure 1(a) depicts the conceptual scheme of the experiment. The single-cycle pulses are set at a variable delay $\Delta \mathrm{t}$ by a broadband dispersion-balanced Mach-Zehnder interferometer and then focused tightly onto the nanocircuit containing a single plasmonic bowtie antenna. The Au optical antenna features a $6 \mathrm{~nm}$ open junction and was fabricated via electron beam lithography (inset in Fig. 1(a)). Under a quasi-static electric bias, this structure exhibits a tunneling-like current-voltage (I-V) characteristic that is antisymmetric and highly nonlinear. The bowtie design allows for a sub-wavelength concentration of the electric field of the laser pulse into the gap while at the same time preserving its single-cycle character owing to the strong radiation damping [36].

a)

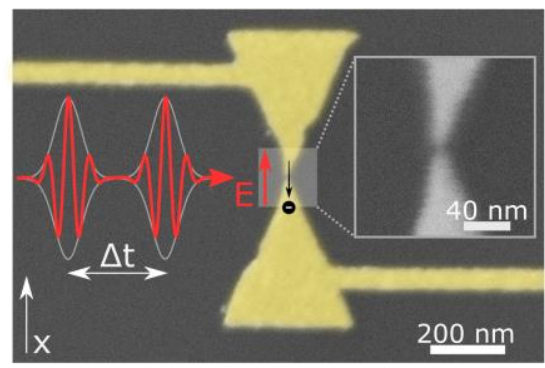

b)

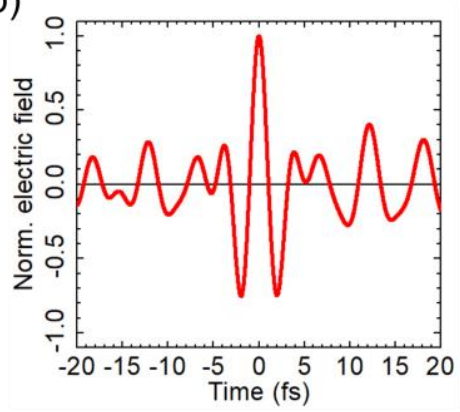

C)

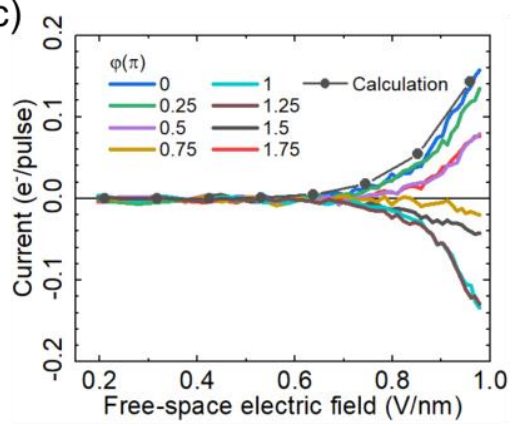

Fig. 1(a) Conceptual sketch of the experiment with two single-cycle light pulses delayed by a time $\Delta t$ and focused on the nanocircuit. The CEP of the biasing pulses can be fully controlled with high precision. The image includes a scanning electron micrograph of the Au optical antenna with electrical leads on a fused-silica substrate and a zoom into the gap region (inset). The positive direction of the electric field is given by the red arrow. (b) Temporal profile of the optical field when set to a cosine phase in free space (CEP=0), as characterized by 2D spectral shearing interferometry [12]. (c) Dependence of the pulse-averaged current induced by the electric field of light as a function of its amplitude and CEP. The current is modulated sinusoidally with a phase $\varphi$ that is directly related to the far-field CEP of the driving pulse with a relative shift of 
approximately $\pi / 2$, as assessed by the numerical calculations. The dotted line reports the TDDFT result obtained for the conditions corresponding to the maximum electron transfer.

A customs-designed Er:fiber laser system [1,10] operates at a repetition rate of $80 \mathrm{MHz}$ and generates 4.2-fs pulses from a tailored supercontinuum spanning from $800 \mathrm{~nm}$ to $2200 \mathrm{~nm}$ generated in a highly nonlinear germanosilicate fiber. After compression, the optical transients have a full-width-at-half-maximum (FWHM) duration that corresponds to a single cycle of the carrier wavelength at $1250 \mathrm{~nm}$. Such single-cycle pulses are ideal for driving ultrafast transport experiments that exploit a non-perturbative phenomenon such as field emission [16]. The temporal profile of the pulses is characterized in Fig. 1(b). A passive phase-locking scheme [11] is implemented in a way that allows arbitrary control of the carrier-envelope phase without affecting the temporal duration of the pulse [16]. These optical transients provide a strong and ultrafast bias for direct and coherent driving of electronic transport across the junction. Due to the strong nonlinearity of the I-V characteristic, an optically-induced symmetry break occurs in the electric transport which leads to a net current that solely depends on the carrier-envelope phase of the driving pulse. Consequently, when sweeping the carrier-envelope phase over a range of $\pi$, the integral current ranges from a maximum, crosses zero and finally completely reverses its direction.

For the autocorrelation measurements, the current generated at the nanojunction of the circuit is recorded as a function of both the delay between the two pulse replicas and their carrier-envelope phase. These measurements were performed in air and at room temperature over several minutes. They demonstrate excellent reproducibility and stability of the nanocircuit, as indicated by the traces depicted in figure 1 (c) that report the current measured as a function of the optical field amplitude of the single-cycle pulses. This dataset was acquired at different relative CEP of the driving pulses on a single sample. Note that only the relative CEP can be defined experimentally. Therefore, $\varphi$ isthe phase of the current and it is set as 0 at the maximum of the sinusoidal modulation in figure 1 (c). At perfect temporal overlap between the two pulses (i.e. delay $\Delta t=0 \mathrm{fs}$, equivalent to a single pulse), the average optical power amounts to $1.7 \mathrm{~mW}$, still corresponding to a minute driving pulse energy of $21 \mathrm{pJ}$ and a peak field of $1 \mathrm{~V} / \mathrm{nm}$ at the sample position within the microscope (1.5 $\mu \mathrm{m}$ spot size). The experimental data obtained with a single pulse have been reproduced with our TDDFT calculations allowing to retrieve the amplitude of the electric field in the gap, as well as to identify the absolute value of the CEP that provides the maximum positive current (also see the SI). 

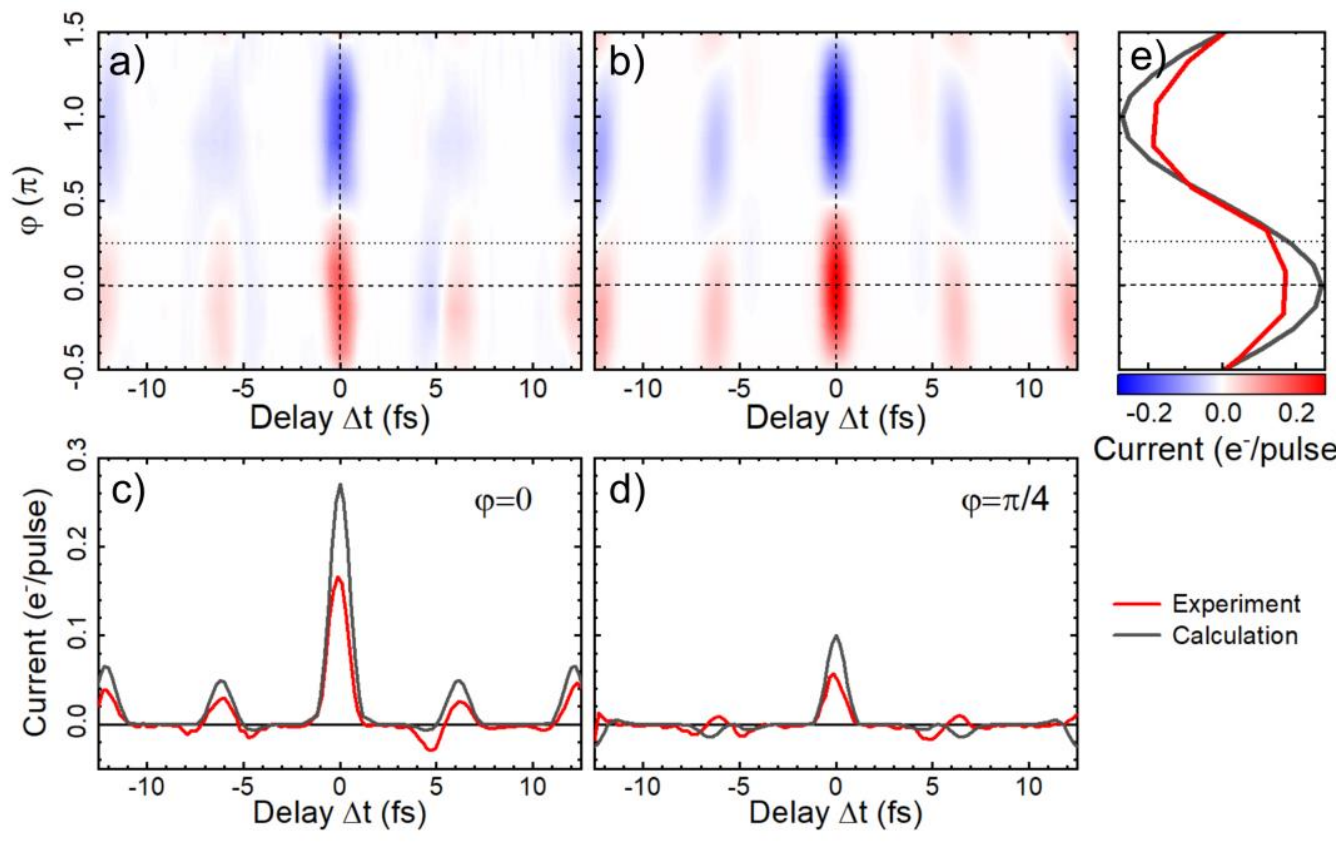

Fig. 2(a) Colour map of the two-dimensional interferometric autocorrelation acquired as a function of temporal delay and CEP of the two driving pulses. The value of $\varphi=0$ is assigned to the carrier-envelope phase that provides maximally positive current. (b) Theoretical interferometric autocorrelation acquired as a function of temporal delay and CEP of the two driving pulses. (c) Interferometric autocorrelation (red line) of two identical single-cycle pulses with CEP set to deliver the maximum peak current using the current driven at the nanogap as nonlinear signal (dashed line in (a) and (b)). The extreme order of nonlinearity is underlined by the absence of signal when the maxima of the field do not overlap at the periodicity of the carrier wavelength. The width of the central peak amounts to approximately $1 \mathrm{fs}$. The graph is compared with the equivalent trace derived from the calculations (black line). (d) same as (c) but acquired at $\varphi=\pi / 4$ (dotted line in (a) and (b)). (e) Vertical cut of the 2D colour map at $\Delta t=0 \mathrm{fs}$, i.e. equivalent to single pulse illuminating the nanogap. The sinusoidal modulation of the current for experiment (red line) and theory (black line) is shown.

Figure 2(a) presents the full 2D map of the interferometric autocorrelation that depicts the nonlinear ultrafast current, with its amplitude colour coded in amplitude from blue to red, driven by a pulse pair as a function of their relative delay and CEP. This measurement allows an insight into the complex non-perturbative phenomenon of field emission in a nanogap since it contains information that goes beyond the standard one-dimensional fringe pattern acquired as a function of the temporal delay between the pulses. Specifically, the colour map contains one additional degree of freedom on the ordinate: the modulation of the current that is accessible by varying the CEP encoded in the single-cycle optical fields. In Fig. 2(b) the theoretical 2D autocorrelation map shows an excellent qualitative agreement with the experimental one (Fig. 2(a)). The comparison between experiment and calculations is presented in Fig. 2(c) and Fig. 2(d) which include a cut of the map at a fixed value of the CEP. This information can be directly compared to a standard interferometric autocorrelation measurement based on optical nonlinear signals. The results 
in figure 2(e) display the dependence of the electron transfer on the CEP at zero time delay, demonstrating full CEP control of the electron currents with a single optical pulse.

From this dataset, it becomes clear that the interferometric autocorrelation originates from a highly non-perturbative process operating on a sub-femtosecond time scale. The interference maxima are significantly shorter than an optical half-cycle of the driving field, featuring a FWHM of approximately $1 \mathrm{fs}$. In addition, the autocorrelation signal completely vanishes between the constructive maxima and the amplitude of the side peaks is strongly reduced with respect to the situation of perfect overlap. Importantly, these findings clearly demonstrate that the electronic transport in the junction is fully coherent with the driving pulses and that the whole current dynamics can be controlled on the timescales shorter than a half-cycle of the driving field.

To gain microscopic insight into the spatio-temporal electron dynamics induced by the optical field in our nanodevice, we carry out first-principles TDDFT simulations. While a full quantum calculation is out of reach for mesoscopic objects like the Au bowties used in the experiments, the main physics can be captured with the reduced system sketched in figure 3(a). Indeed, the plasmonic field enhancement strongly confines the region of optical field emission and electron transport to the junction, as follows from the snapshots of the calculated electron current density depicted in figure 3(b). In detail, we model the plasmonic gap as two infinite parallel Au cylinders described within the free electron (jellium) model. The cylinder radii $R_{c}$ and their separation distance $d_{g}$ are adapted to the shape of the antenna as visualized by the SEM images $\left(R_{c}=5 n m\right.$ and $\left.d_{g}=6 n m\right)$. The time trace of the electric field in the gap of the cylinder in the TDDFT calculations is adjusted to the one obtained from classical Maxwell's equations for the actual bowtie antenna. The absolute value of the field is calibrated with respect to the experimental dependence of the current on the pulse energy (see calibration points in figure 1(c) plotted as grey line with dots).

The effect of the plasmonic resonance in the near-field at the gap region of the bowtie nanoantenna may be seen in Fig. 3(c). The far-field transient (red line) of a pulse with CEP $=0$ in free space becomes distorted within the antenna gap (green line). The induced field in the junctions is enhanced by approximately 85 times with respect to the incident pulse. In addition, a phase shift by $\mathrm{CEP}_{\text {gap }} \approx 0.35 \pi$ occurs with respect to the envelope. The plasmon resonance of the nanoantenna results in a pronounced few-cycle ringing of the near-field with a characteristic phase shift of $\pi / 2$. 
a)

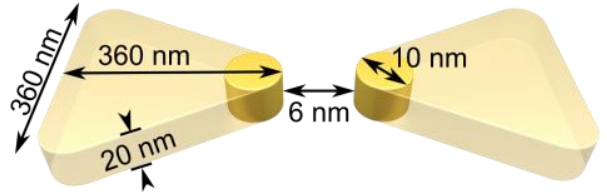

b)

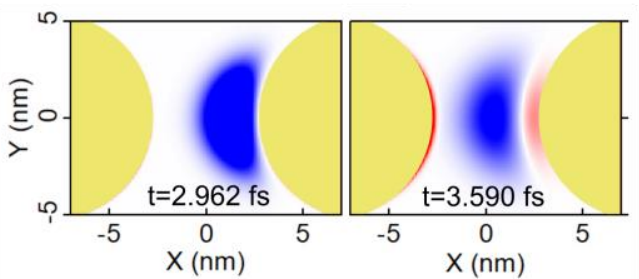

c)

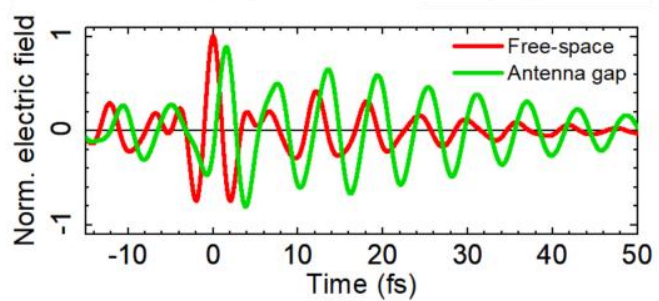

d)

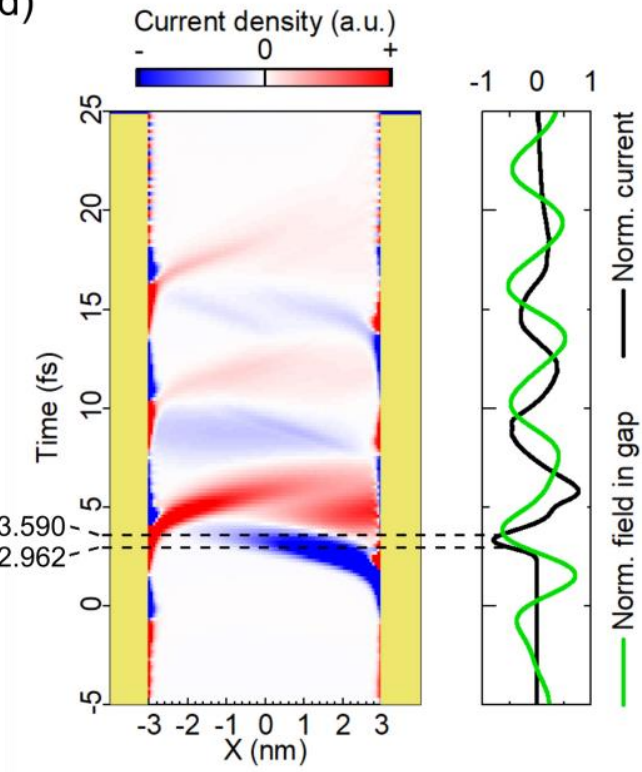

Fig. 3(a) Conceptual sketch of the antenna geometry employed for the calculations. The cylinders reproducing the morphology of the antenna apexes are used in the TDDFT calculations of the optical field emission while the plasmonic resonance corresponding to the whole triangular antenna gap is captured by solving the classical Maxwell equation. (b) Snapshots of the electron current density within the gap at two different times $(t=0$ is set at the maximum of the free-space optical field). The dashed lines in panel $d$ corresponds to the times at which the snapshots are captured. (c) Electric field profile of the far-field illumination of a pulse with $C E P=0$ (red) and corresponding electric field profile calculated in the centre of the gap (green). The fields in the antenna gap are scaled by a factor of $1 / 100$. (d) Colour map of the electron current along the dimer axis, calculated as a function of time for far-field illumination with $\mathrm{CEP}=\mathbf{0}$. (right) corresponding electric field in the gap (green) and integrated electron current across the gap central plane (black).

The enhanced electric field in the junction region drives the optical field emission and thus the electron flow through the gap, as illustrated in figure $3(\mathrm{~d})$. For an illumination with a CEP $=0$ pulse in free space, the antenna resonance shapes the temporal profile of the transient to become almost sine-like in the near-field. This effect, combined with the temporal dynamics of the electrons within the gap, leads to two opposite electronic streams - which are produced by the central cycle of the pulse and which almost completely cancel out each other. The net electron transport through the gap is therefore close to zero in this situation. The entire dynamics is repeated by the following cycles at strongly reduced magnitudes because of the progressive decay of the induced near-field. Paradoxically, the maximum positive current is produced when the pulse in the far field is closer to a sine-like profile with a corresponding CEPgap within the antenna near-field that approaches $\pi$ (this case displays a simpler dynamics of the electrons within the gap. The respective colour map of the trajectories can be found in the supporting information as fig. S6). 
The finite propagation time of the photoemitted electrons through the antenna gap explains an additional offset of the oscillation of the current through the middle of the junction with respect to that of the field in the gap. Noteworthy, for the present geometry, the trajectories followed by the electrons differ from what is typically observed in the case of metal tips. For optical field emission from nanotips or individual plasmonic nanoobjects, the fast spatial decay of the electric near-fields reduces the quiver motion of emitted electrons $[2,5,20,24,25]$. However, contrary to the case of a single nanoparticle, the electric field in a metallic gap is rather uniform. This fact renders the dynamics of electron transport to be strongly affected by the quiver motion as illustrated in Fig. 3(d). In detail, the early electrons are ejected from the right surface by the positive field during the first half-period of the pulse. The initial acceleration within the gap is sketched in the left snapshot of figure 3b. Since the driving pulse is sine-like, the polarity of the field changes when the electrons are still within the gap. This situation leads to a strong deceleration of electrons at the tail of the bunch and to simultaneous emission of a current stream with opposite direction. This situation is depicted in the right snapshot of Fig. 3(b) where red regions corresponding to positive current density appear within the gap. Figure 3(d) further follows the dynamics with a complete reversal of the sign of the electron current density upon the change of polarity within the second half-cycle.

In the present case the amplitude of the quiver motion $X_{Q}=E_{g} / \omega^{2}\left(E_{g}\right.$ in $\mathrm{V} / \mathrm{nm}$ is the maximum value of the field in the gap, and $\omega$ is the central frequency of the pulse), is smaller than the size of the gap, thus enabling coherent motion of the ejected electrons. This aspect has a direct consequence on the CEP conditions for the maximum electron transport to occur. Using classical equations of motion, the final momentum of the electron emitted at the instant of time $\tau$ is proportional to the instantaneous vector potential of the field in the gap: $p(t=\infty) \propto A(\tau)$. Thus, despite the fact that the strongest electron emission is produced at the maximum of the field in the gap, the instantaneous value of the vector potential might be zero. The emitted electrons will not cross the junction since $X_{Q}<d_{g}$. We calculate that the maximum electron transfer is reached for the effective CEPgap $=0.84 \pi$ (i.e. for CEP $=0.49 \pi$ of the incident pulse in the far field). Therefore, the match between the measured and calculated sinusoidal modulation of the current at delay $=0 \mathrm{fs}$ in figure 2(e) allows us to unambiguously determine the absolute value of the free-space CEP in the experiment. Along with the possibility to determine the CEP of the experimental transient, the remarkable agreement between the modelled and experimental results also reveals the role of the plasmon mode of the nanoantenna: the secondary maxima in the experimental autocorrelation plot in figure 2(c) emerge owing to the resonant enhancement of the near-fields at large time delays. In fact, in the ideal case of a flat antenna response, the autocorrelation signal obtained with TDDFT is zero at every time delay except for the central cycle.

In both measured and calculated data, the CEP-dependence of the secondary maxima is slightly phase shifted with respect to the main peak. This result stems from: (i) the non-gaussian, asymmetric profile of the driving pulse for which the slowly varying envelope approximation is not valid (i.e. the carrier cannot be considered monochromatic) 
and thus may be affected by some residual chirp; (ii) the resonance effect of the plasmonic antenna that shapes the field within the gap and defines the quiver motion of electrons emitted at different instants of time. This aspect becomes even more clear at a value of $\varphi=\pi / 4$ in the autocorrelation maps of figure $2(a)$ and (b). The corresponding data are plotted in figure 2 (d) as red and black lines for experiment and theory, respectively. The signs of the secondary maxima are reversed with respect to the ones of the main peak at $\Delta t=0$. Altogether, the high nonlinearity of the optical field emission allows using our device as a sensitive probe of the dynamics of the near fields in plasmonic gaps.

In conclusion, we have performed interferometric autocorrelation measurements with single-cycle pulses that exploit the electric currents coherently driven at the gap of a single nanodevice. These measurements operate in an extremely nonlinear regime that is achieved even at minute $\mathrm{pJ}$ pulse energies. A complex dynamics for the fielddriven transport of the electrons emerges in free space that offsets the carrier-envelope phase of the driving field. Remarkably, the extreme temporal resolution that we achieve in performing current autocorrelation demonstrates that we can control the transfer of individual electrons between two metallic nanocontacts and access the evolution of plasmonic fields in the nanogap at a subcycle time scale. In such a scenario, the photoemitted electrons experience quiver motion, which responds to the complex structure of the fields induced in the gap, resulting in a shift of the phase at which the maximum current is obtained. Therefore, tracing and dissecting an ultrashort photocurrent in a plasmonic nanogap is found to be crucial for the proper design of integrated optoelectronic devices that operate in the single-electron regime at optical frequencies. In addition, the fundamental understanding of electronic dynamics at the nanoscale achieved in this work will allow to target fundamental experiments where the Coulomb interaction between electrons becomes important owing to the subcycle confinement of transport to truly atomic time and length scales.

\section{Methods}

Methods and any associated references are available in the online version of the paper.

\section{Source of control transients}

The elements of the Er:fiber laser system providing the ultrashort optical control pulses exploited in our experiment are described in detail in Ref. 16. Here, for the first time, single-cycle near-infrared transients that are passively phase locked were generated at a repetition rate as high as $80 \mathrm{MHz}$ by combining super-octave-spanning coherent synthesis with elimination of the carrierenvelope phase slip via difference frequency mixing and re-amplification. The present implementation of the system is even superior to our earlier results in terms of pulse duration [1]. The laser system also offers precise CEP control by placing the pair of fused-silica wedges before the last Er:fiber amplifier and final compression. The minimum duration is achieved by the independent compression of soliton and dispersive wave (with a three-prism setup) before coherent combination of the two spectral components produced by a highly nonlinear fiber. 
For a full temporal reconstruction of the pulse we performed a direct characterization via two-dimensional spectral shearing interferometry. This method allows direct reconstruction of the electric field profile, i.e. the amplitude and the phase of the electromagnetic wave. It is particularly suited for super-octave-spanning spectra. By fine tuning the dispersion via an SF10 prism pair, we obtain an almost flat phase over the entire spectrum. This result corresponds to a pulse duration of $4.2 \mathrm{fs}$ and 1 optical cycle at the central wavelength of $1250 \mathrm{~nm}$. The complete characterization is included in the supplementary material.

\section{Antenna fabrication and characterization}

The nanostructured electronic circuit equipped with a plasmonic antenna was fabricated by electron beam lithography. A fused silica window was spincoated with a $60 \mathrm{~nm}$ layer of PMMA (950k) resist. Heating the substrate above $100^{\circ} \mathrm{C}$ before deposition evaporates residual water on the surface. An $8 \mathrm{~nm}$-thick thermally evaporated layer of aluminum provided the necessary electric conduction to avoid distortion due to charging effects during the exposure. The structures were exposed at an area dose of 400 $\mathrm{pC} / \mathrm{cm}^{2}$ at $10 \mathrm{kV}$. After the exposure the aluminum layer was removed in a bath of sodium hydroxide followed by a rinse in deionized water. Development of the resist was carried out in a mixture of methyl isobutyl ketone (MIBK), methyl ethyl ketone (MEK) and isopropanol (IPA) in a volume ratio of 100:6:300. The gold nanostructures were patterned in a subsequent evaporation process in which $18 \mathrm{~nm}$ of gold were evaporated on top of a $2 \mathrm{~nm}$ chromium adhesion layer followed by a lift-off process in a bath of acetone. The electronic interface between the nanostructures and the detection electronics was provided by a custom-made circuit board into which the fused silica substrates were integrated and connected by wire bonding.

\section{TD-DFT}

The dynamics of the electron density induced by a laser pulse has been obtained by solving the time-dependent Kohn-Sham (KS) equations [17].

$$
i \partial_{t} \psi_{k}(\vec{r}, t)=\left\{T+V_{A u}+V_{x c}(n)+V_{H}(n)+V^{e x t}(t)\right\} \psi_{k}(\vec{r}, t)
$$

This equation describes the evolution of the quasiparticle Kohn-Sham (KS) orbitals $\psi_{j}$ under the action of an external potential $V^{e x t}$. The incident laser pulse is treated within the dipole approximation. In the Coulomb gauge the external potential is given by $V^{e x t}=E^{e x t}(t) x$, where $E^{\text {ext }}(t)$ describes the external field, and the dimer $x$-axis is defined in Fig. 3a. In Eq. (1) $T$ is the kinetic energy operator, $V_{A u}$ describes the electron interaction with atomic cores, $V_{x C}(n)$ is the exchange-correlation potential treated within the adiabatic local density approximation [17] with exchange-correlation kernel of Gunnarson and Lundquist [18], and $V_{H}(n)$ is the Hartree potential. The time-dependent electronic density of the system is given by $n(\vec{r}, t)=$ $\sum_{k=o c c} \psi_{k}(\vec{r}, t)$ where the summation runs over the occupied KS orbitals. The initial conditions $\psi_{k}\left(t_{0}\right)$ are given by the KS orbitals of the ground state system, obtained from the ground-state density functional theory (DFT) calculation,

$$
\underbrace{\left\{T+V_{A u}+V_{x c}\left(n_{0}\right)+V_{H}\left(n_{0}\right)\right\}}_{H_{0}} \psi_{k}(\vec{r}, t=0)=E_{k} \psi_{k}\left(\vec{r}, t_{0}\right),
$$

where the Hamiltonian $H_{0}$ depends on the ground-state density $n_{0}(\vec{r})=\sum_{k=o c c} \psi_{k}\left(\vec{r}, t_{0}\right)$ via the exchange-correlation and Hartree potentials. Consistent with TDDFT, the exchange-correlation kernel of Gunnarson and Lundquist is used for $V_{x c}\left(n_{0}\right)$. 
Considering the dimer of two infinite cylindrical nanowires instead of the bowtie antenna brings the system within the reach of quantum calculations if the free electron (jellium) description of metal [19] is used. Within the jellium model (JM) the ionic cores at the gold lattice sites are replaced by the uniform positive background charge of the density $n+=\left[\frac{4 \pi}{3} r_{S}^{3}\right]^{-1}$ We use $r_{s}=3$ ao characteristic for the valence electron density of gold ( $a_{0}=0.053 \mathrm{~nm}$ stands for the Bohr radius). At the price of losing atomistic details, this approach allows to follow the dynamics of the valence electrons of gold involved in the screening, optical field emission, and electron transport $[3,20,21,22]$. Note also that the JM has been successfully used in the context of plasmonics allowing theoretical prediction of quantum effects later confirmed experimentally [23].

The model cylindrical nanowire dimer reproduces well the optical field electron emission and electron transport in the gap of experimental bowtie antenna. However, the exact description of the electric field induced in the gap region, including the macroscopic effect of the actual triangular dimer, is of paramount importance for capturing the details of the electron transport. To this end we solve the classical Maxwell's equations, and we calculate the field in the gap $E_{g}(t)$ for the actual silica-supported bowtie nanoantenna subjected to the experimental incident pulse profile $E^{\text {ext }}(t)$. (As a reference we use the field $E_{g}(t)$ in the middle of the junction). In the TDDFT calculations, $E^{e x t}(t)$ is then set in such a way that the self-consistent field in the gap equals to $E_{g}(t)$. The absolute value of the electric field strength used in the TDDFT calculations has been set such that the experimental data obtained with $\Delta t=0$ and the CEP corresponding to the maximum electron transfer are reproduced as shown in Fig. 1 . We calculated that for a transfer of 0.2 electrons per pulse the field in the gap is of the order of $10 \mathrm{~V} / \mathrm{nm}$. This implies an 11 -fold enhancement of the free-space electric field which is essentially lower than the theoretical estimate based on the solution of the classical Maxwell equations. However, it has to be considered as an effective value accounting for the experimental uncertainties, as well as for the model geometry used in the TDDFT study, and possible nonlinear effects leading to a strong reduction of the field enhancement as compared to the predictions of the classical linear theory [35].

Provided the time-dependent solutions of the KS equations $\psi_{k}(\vec{r}, t)$, one can obtain the time-dependent current density

$$
\vec{J}(\vec{r}, t)=-i \sum_{k=o c c} \frac{1}{2}\left\{\psi_{k}(\vec{r}, t) \nabla \psi_{k}(\vec{r}, t)-\psi_{k}(\vec{r}, t) \nabla \psi_{k}(\vec{r}, t)\right\}
$$

and the total electron current per unit height through the middle of the gap

$$
I(t)=\left.\int d y \hat{e}_{x} \vec{\jmath}(\vec{r}, t)\right|_{x=0}
$$

The theoretical prediction for the number of electrons transferred across the gap of the bow tie nanoantenna is then given by $N=h \int_{t_{0}}^{T} d t I(t)$, where $\mathrm{h}=20 \mathrm{~nm}$ is the height of the nanoantenna. The final propagation time is taken large enough so that the electric field becomes small and the electron transfer stops. 


\section{References}

1. Krauss, G. et al. Synthesis of a single cycle of light with compact erbium-doped fibre technology. Nat. Photonics 4, 33-36 (2010).

2. Herink, G., Solli, D., Gulde, M. \& Ropers, C. Field-driven photoemission from nanostructures quenches the quiver motion. Nature 483, 190-193 (2012).

3. Lemell, C., Tong, X., Krausz, F. \& Burgdörfer, J. Electron Emission from Metal Surfaces by Ultrashort Pulses: Determination 3of the Carrier-Envelope Phase. Phys. Rev. Lett. 90, 076403 (2003).

4. Dombi, P., Krausz, F. \& Farkas, G. Ultrafast dynamics and carrier-envelope phase sensitivity of multiphoton photoemission from metal surfaces. J. Mod. Opt. 53, 163-172 (2006).

5. Krüger, M., Schenk, M. \& Hommelhoff, P. Attosecond control of electrons emitted from a nanoscale metal tip. Nature 475, 7881 (2011).

6. Schiffrin, A. et al. Optical-field-induced current in dielectrics. Nature 493, 70-74 (2012).

7. Piglosiewicz, B. et al. Carrier-envelope phase effects on the strong-field photoemission of electrons from metallic nanostructures. Nat. Photonics 8, 37-42 (2013).

8. Paasch-Colberg, T. et al. Solid-state light-phase detector. Nat. Photonics 8, 214-218 (2014).

9. Cocker, T. L. et al. An ultrafast terahertz scanning tunneling microscope. Nat. Photonics 7, 620-623 (2013).

10. Brida, D., Krauss, G., Sell, A. \& Leitenstorfer, A. Ultrabroadband Er:fiber lasers. Laser Photon. Rev. 8, 409-428 (2014).

11. Krauss, G. et al. All-passive phase locking of a compact Er:fiber laser system. Opt. Lett. 36, 540-542 (2011).

12. Birge, J. R., Ell, R. \& Kärtner, F. X. Two-dimensional spectral shearing interferometry for few-cycle pulse characterization. Opt. Lett. 31, 2063-2065 (2006).

13. Savage, K. et al. Revealing the quantum regime in tunnelling plasmonics. Nature 491, 574-577 (2012).

14. Esteban, R. et al., A classical treatment of optical tunneling in plasmonic gaps: extending the quantum corrected model to practical situations. Faraday Discuss. 178, 151-183 (2015).

15. Wan, Y., Wubs, M. \& Mortensen, N. A., Projected Dipole Model for Quantum Plasmonics. Phys. Rev. Lett. 115, 137403 (2015).

16. Rybka, T. et al. Sub-cycle optical phase control of nanotunnelling in the single-electron regime. Nat. Photonics 10, 667-670 (2016).

17. Marques, M. A. L. \& Gross, E. K. U. Time-dependent density functional theory. Annu. Rev. Phys. Chem. 55, 427-455 (2004).

18. Gunnarsson, O. \& Lundqvist, B. I. Exchange and correlation in atoms, molecules, and solids by the spin-density-functional formalism. Phys. Rev. B 13, 4274-4298 (1976)

19. Lang, N.D. \& Kohn W. Theory of metal surfaces: work function. Phys. Rev. B 3, 1215-1223 (1971). 
20. Krüger, M., Lemell, C., Wachter, G., Burgdörfer, J. \& Hommelhoff, P. Attosecond physics phenomena at nanometric tips. J. Phys. B 51, 172001 (2018).

21. Gao, C.-Z. et al. Strong-field effects in the photoemission spectrum of the C60 fullerene. Phys. Rev. A 93, 022506 (2016).

22. Nguyen, H.S., Bandrauk, A. D. \& Ullrich, C. A. Asymmetry of above-threshold ionization of metal clusters in two-color laser fields: a time-dependent density-functional study. Phys. Rev. A 69, 063415 (2004).

23. Zhu, W. el al. Quantum mechanical effects in plasmonic structures with subnanometre gaps. Nat. Commun. 7, 11495 (2016).

24. Echternkamp, K. E. et al. Strong-field photoemission in nanotip near-fields: from quiver to sub-cycle electron dynamics. Appl. Phys. B 122, 80 (2016).

25. Dombi, P. et al. Ultrafast Strong-Field Photoemission from Plasmonic Nanoparticles. Nano Lett. 13, 674-678 (2013).

26. Aguirregabiria, G. et al. Dynamics of electron-emission currents in plasmonic gaps induced by strong fields. Faraday Discuss. Preprint at http://doi.org/10.1039/C8FD00158H (2018).

27. Cocker, T. L., Peller, D., Yu, P., Repp, J. \& Huber, R. Tracking the ultrafast motion of a single molecule by femtosecond orbital imaging. Nature 539, 263-267 (2016)

28. Ahn, B. et al. Attosecond-controlled photoemission from metal nanowire tips in the few-electron regime. APL Photonics $\mathbf{2}$, 036104 (2017)

29. Vogelsang, J., Hergert, G., Wang, D., Groß, P. \& Lienau, C. Observing charge separation in nanoantennas via ultrafast pointprojection electron microscopy. Light-Sci. Appl. 7, 4-11 (2018)

30. Jelic, V. et al. Ultrafast terahertz control of extreme tunnel currents through single atoms on a silicon surface. Nat. Phys. 13, 591-598 (2017)

31. Yoshioka, K. et al. Tailoring Single-Cycle Near Field in a Tunnel Junction with Carrier-Envelope Phase-Controlled Terahertz Electric Fields. Nano Lett. 18, 5198-5204 (2018)

32. Yoshioka, K., Katayama, I., Minami, Y., Kitajima, M. \& Yoshida, S. (2016). Real-space coherent manipulation of electrons in a single tunnel junction by single-cycle terahertz electric fields. Nat. Photonics 10, 762-765 (2016)

33. Putnam, W. P., Hobbs, R. G., Keathley, P. D., Berggren, K. K. \& Kärtner, F. X. Optical-field-controlled photoemission from plasmonic nanoparticles. Nat. Phys. 13, 335-339 (2017)

34. Rácz, P. et al. Measurement of Nanoplasmonic Field Enhancement with Ultrafast Photoemission. Nano Lett. 17, 1181-1186 (2017)

35. Marinica, D.C., Kazansky, A. K., Nordlander, P., Aizpurua, J. \& Borisov, A. G. Quantum Plasmonics: Nonlinear Effects in the Field Enhancement of a Plasmonic Nanoparticle Dimer. Nano Lett 12, 1333-1339 (2012) 


\section{Acknowledgements}

DB and AL acknowledge support of the Deutsche Forschungsgemeinschaft through the Emmy Noether programme and the collaborative research center SFB 767. GA acknowledges project PI2017-30 of the Departmento de Educación, Política Lingüística y Cultura of the Basque Government, and GA and JA acknowledge funding from project FIS2016-80174-P of the Spanish Ministry of Science, Innovation and Universities MICINN.

\section{Author Contributions}

$J A, A G B, A L$ and $D B$ conceived the project. $A L$ and $D B$ supervised the experimental activity. $M L, F R$ and $T R$ fabricated the nanostructures, developed the setup and performed the measurements. JA and AGB coordinated the theoretical modeling. GA, D-CM and AGB developed the theory simulations. All authors contributed to the discussion of the data and to the manuscript.

\section{Additional information}

Correspondence and requests for materials should be addressed to Daniele Brida: daniele.brida@uni.lu

\section{Competing financial interests}

The authors declare no competing financial interests. 